\title{
How Patient-Pharmacist Communication Using the Drug Profile Book Relates to Patient's Behavior regarding Its Use
}

\author{
Masaki Shoji, ${ }^{a}$ Kentaro Iwade, ${ }^{b}$ Keiko Fujii, ${ }^{b}$ Miyuki Hirota, ${ }^{b}$ Akira Kanou, ${ }^{b}$ \\ Mami Moriya, ${ }^{b}$ Masaki Ishii, ${ }^{b}$ Shizuka Shimoji, ${ }^{b}$ Mitsuko Onda, ${ }^{*, a}$ and Yukio Arakawa ${ }^{a}$ \\ ${ }^{a}$ Clinical Laboratory of Practical Pharmacy Osaka University of Pharmaceutical Sciences; 4-20-1 Nasahara, Takatsuki, \\ Osaka 569-1094, Japan: and ${ }^{b}$ Saera Pharmacy; 2-2-5 Honmachi, Chuou-ku, Osaka 541-0053, Japan.
}

(Received April 6, 2016; Accepted June 8, 2016)

\begin{abstract}
This survey aimed to examine how patient-pharmacist communication using the drug profile book relates patient's behavior regarding its use. Among patients who visited one of the five pharmacies during the 4 months between July and October of 2013, 245 patients who had been prescribed antihypertensives were asked to complete a questionnaire. Items included patient attributes, whether the patient thought the drug profile book was useful to them ("sense of utility"), whether the patient has ever been questioned by a pharmacist while showing the drug profile book ("experience of being questioned by a pharmacist while showing the drug profile book"), and whether the patient has ever shown the drug profile book to the physician ("experience of showing the drug profile book to the physician"). In addition, pharmacists counted the frequency of patients bringing the drug profile book, and if so, the frequency of the sticker affix during the last 5 visits. $34.3 \%$ of responding patients answered that they had the "experience of being questioned while showing the drug profile book". Response rates of "frequency of bringing the drug profile book", "sense of utility", and "experience of showing the drug profile book to the physician" in the group with "experience of being questioned while showing the drug profile book" were significantly higher than those in the group without such experience. This survey indicated that experience of being questioned by a pharmacist while showing the drug profile book related patient's behavior regarding its use.
\end{abstract}

Key words_ drug profile book; community pharmacy; pharmacist; communication; hypertension

\section{INTRODUCTION}

In Japan, separation of dispensary from medical practice was established in 1871 with the decision by the government to adopt the German medical system. However, because there were very few pharmacists at the time, and because there was a long-held national familiarity with the traditions of Chinese herbal remedies, the system's development was slow. This system did not gain momentum until the prescription fee was raised to 50 points ( $¥ 500)$ in 1974 . Since then, there has been gradual development. ${ }^{1)}$ Even now, however, some people question separation of dispensary from medical practice, wondering whether it is really to the benefit of the Japanese people. ${ }^{2)}$ Currently, while 67 $\%$ of total prescriptions (as of 2013) are dispensed at pharmacies, the system, which has been promoted under the guidance of the Ministry of Health, Labour and Welfare, is faced with the challenge of a change in focus from quantity to quality. To enhance the quality, obtaining comprehensive drug information

\footnotetext{
*e-mail: onda @gly.oups.ac.jp
}

of the patient is necessary for pharmacists to check drug interaction by reference to other concomitant drugs and previous information. ${ }^{3)}$ In Japan, there is a medical medium to record patients' drug information comprehensively called "drug profile book".

The use of the drug profile book is a Japan-specific medical medium that has been in place since 1983 with the aim of avoiding medical incidents. The drug profile book is about the size of a postcard and serves as a medium for recording drug administration history. With each patient's agreement, the pharmacist issues him or her with a drug profile book in which information is accumulated about the drugs the patient has been prescribed by affixing "the sticker" in the book, mainly carrying details about prescriptions dispensed by pharmacies (Fig. 1). The patient brings along the drug profile book when he or she attends a medical facility and shows it to the health professionals. The drug profile book, then, fulfills the role of preventing duplicate medication or drug interaction. If the patient has forgotten to bring the drug profile book, the pharmacy often gives him or her the sticker and asks him or her to affix it in later. 


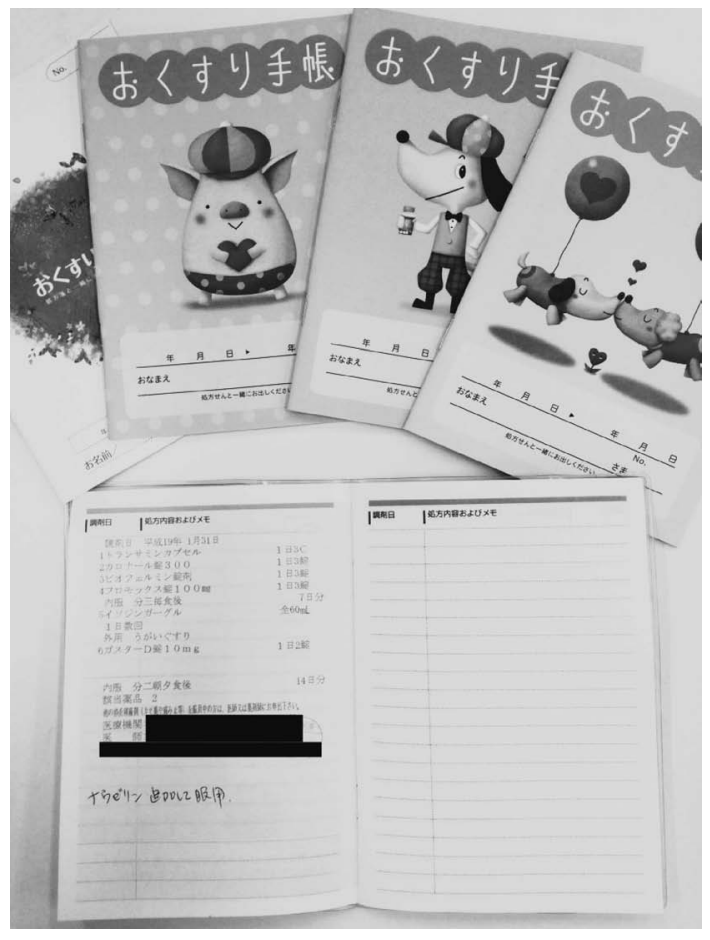

Fig. 1. The Cover of the Drug Profile Book and the Sticker Affixed Inside It

Since dispensing fee revision in April 2000, the use of the drug profile book was disseminated as an important medium of the main health care system. In the drug profile book, patient information such as history of drug administration, adverse drug reactions, allergy and drug interaction, is recorded. This information plays an important role in preventing duplicate medication or drug interaction in patients visiting multiple medical facilities. Following the Great East Japan Earthquake of 2011, the health care infrastructure was temporarily paralyzed by tsunami damage, and patient information, including official records, was lost. As an analogue medium that did not rely on electricity, the drug profile book could be utilized at that difficult time, facilitating the smooth recommencement of medical treatment. This reconfirmed the importance of the drug profile book as a medical information medium. ${ }^{4)}$ However, problems with the proper use of the drug profile book have been pointed out recently; for instance, the sticker has not been affixed, and some patients have received multiple drug profile books from each medical facility. 5,6$)$

In 2013, we conducted a survey to clarify how frequently each patient taking the antihypertensive brought the drug profile book to the pharmacy; how frequently the patient affixed the sticker for the drug profile book; and the correlated factors thereof. The survey indicated that utilization of the drug profile book is related to patient's recognition of its role and its sense of utility. ${ }^{7)}$ Therefore, in this paper, we examine the relationship between patient-pharmacist communication related to the drug profile book at each medication consultation and the patient's behavior regarding its use, based on the database obtained foregoing survey. ${ }^{7)}$

\section{METHODS}

Survey Period and Participants The survey was conducted for the 4 months from July to October in 2013.7) Five pharmacies visited by more than 5 patients per day to receive antihypertensives were selected from among pharmacies located in Osaka, Hyogo, Okayama, Tokyo, and Aichi with cooperation of a company that runs pharmacies and has its headquarters in Osaka. Prior to the survey, survey outline was explained to pharmacists working for these pharmacies. Patients visiting one of these 5 pharmacies and receiving antihypertensives were included in the survey. Patients with dementia, patients with difficulty in filling the questionnaire, first-time patients, and patients who have somebody else receive drug for them were excluded. Based on following 3 reasons, we limited participants to patients who received antihypertensives during this survey: (1) patients who made regular visits to the pharmacy were needed for recording the frequency of bringing the drug profile book and frequency of the sticker affix over time, (2) bias generated by including multiple diseases should be avoided when comparing the efficacy of communication between pharmacists and patients using the drug profile book, and (3) patients with chronic diseases with relatively high morbidity were needed to ensure a sufficient number of patients.

Survey Items Principal survey items include (1) patient attributes (gender, age, number of taken drugs, consultation with plural clinics, and number of complicating diseases), (2) whether the patient thought the drug profile book was useful to them ("sense of utility"), (3) whether the patient has ever shown the drug profile book to the physician, ("experience of showing the drug profile book to the physician"), (4) whether the patient has ever been questioned by a pharmacist while showing the drug profile book ("experience of being questioned by a pharmacist while showing the drug profile book"), (5) 
the number of times the patient has used the sticker ("frequency of the sticker affix") (This included both occasions - when the patient brought the drug profile book along and the pharmacist affixed the sticker and when the patient did not bring the drug profile book and so affixed the sticker himself or herself.), and (6) the number of times the patient has brought the drug profile book with him or her ("frequency of bringing the drug profile book").

Patients were asked to rate sense of utility on a 5point scale (yes, slightly yes, neither of both, slightly no, no) which were scored (yes $=5$, no $=1$ ) .

Frequency of the sticker affix was confirmed by checking the latest 5 visits at the first survey day to verify that the dates of the sticker affix on the drug profile book corresponded to the patient's visits. Only the sticker provided by pharmacies included in this survey. Frequency of bringing the drug profile book was calculated by counting how many times the patient included in this survey brought the drug profile book between the first and the 5th visit. Information regarding (1) patient attributes, (2) sense of utility, (3) experience of showing the drug profile book to the physician, and (4) experience of being questioned by a pharmacist while showing the drug profile book was collected based on the questionnaire given to the patient, and pharmacists were asked to fill out the summary table in an Excel file for (5) frequency of the sticker affix and (6) frequency of bringing the drug profile book.

The patients and the questionnaire sheet in this survey are identical to those in the foregoing survey. ${ }^{7)} \mathrm{By}$ the foregoing survey, we have already obtained the following results. Table 1 shows the patient attributes. To the question of sense of utility, most frequent response was "yes" $(60.4 \%)$, followed by "slightly yes" (24.1\%). To the question of frequency of bringing the drug profile book, 2 times was the most frequent $(24.5 \%)$, while 5 times was $12.2 \%$. Average was 2.3 times. To the question of frequency of the sticker affix, 5 times was the most frequent $(73.5 \%)$, while 0 times was $4.5 \%$. Average was 4.5 times. ${ }^{7)}$

Statistical Analysis Participants were divided into 2 groups: those with or without experience of being questioned by a pharmacist while showing the drug profile book. A Mann-Whitney $U$ test was used to examine the relationship with frequency of bringing the drug profile book, sense of utility, and fre-
Table 1. Patient Attributes

\begin{tabular}{lcc}
\hline \hline Gender $\quad$ Male & 107 \\
& Female & 138 \\
Age (years) [mean (S.D.)] & $68.3(11.1)$ \\
Number of taken drugs [mean (S.D.)] & $4.2(2.6)$ \\
Consultation with plural clinics (Yes) (\%) & 53.7 \\
Number of complicating diseases [mean (S.D.)] & $1.4(0.7)$ \\
\hline
\end{tabular}

quency of the sticker affix. Pearson's $\chi^{2}$ test was used to examine the relationship with experience of showing the drug profile book to the physician. In the same way, the Mann-Whitney $U$ test was used to examine the relationship between i) the degree of "sense of utility" and ii) "experience of showing the drug profile book to the physician". The level of significance was set at $5 \%(0.05)$. PASW Statistics 18 was used for all analyses.

Ethical Consideration This survey was conducted after obtaining approval of the Ethical Review Board of Osaka University of Pharmaceutical Sciences.

\section{RESULTS}

Relationship between the Experience of Being Questioned by a Pharmacist while Showing the Drug Profile Book and Patient Attributes No significant difference in patient attributes was observed between groups with ( 84 patients) and without (148 patients) experience of being questioned by a pharmacist while showing the drug profile book (Table 2) .

Relationship between Patient's Behavior and the Experience of Being Questioned by a Pharmacist while Showing the Drug Profile Book MannWhitney $U$ tests were used to examine the differences in frequency of bringing the drug profile book, frequency of the sticker affix, and sense of utility, between groups with and without the experience of being questioned by a pharmacist while showing the drug profile book. Response rates of "frequency of bringing the drug profile book", "sense of utility" were significantly higher in the group with the experience of being questioned by a pharmacist while showing the drug profile book compared to the group without such experience (Table 3 ).

Pearson's $\chi^{2}$ test was used to examine the difference in distribution of the "experience of showing the drug profile book to the physician" between groups with and without the experience of being questioned by a 
Table 2. Relationship between the Experience of Being Questioned by a Pharmacist while Showing the Drug Profile Book and Patient Attributes

\begin{tabular}{|c|c|c|c|c|c|c|}
\hline & & $\begin{array}{c}\text { Male } \\
(\%)\end{array}$ & $\begin{array}{c}\text { Age } \\
\text { (years) } \\
{[\text { mean (S.D.)] }}\end{array}$ & $\begin{array}{l}\text { Number of } \\
\text { taken drugs } \\
{[\text { mean (S.D.) }]}\end{array}$ & $\begin{array}{l}\text { Consultation } \\
\text { with } \\
\text { plural clinics } \\
\text { (Yes) (\%) }\end{array}$ & $\begin{array}{c}\text { Number of } \\
\text { complicating } \\
\text { diseases } \\
{[\text { mean (S.D.) }]}\end{array}$ \\
\hline \multirow{2}{*}{$\begin{array}{l}\text { Have you ever been questioned by a } \\
\text { pharmacist while showing your drug } \\
\text { profile book? }\end{array}$} & Yes $\quad(n=84)$ & 41.7 & $69.0(9.8)$ & $4.3(2.6)$ & 41.1 & $1.5(0.8)$ \\
\hline & No $(n=148)$ & 46.6 & $66.7(11.4)$ & $4.0(2.5)$ & 58.9 & $1.4(0.7)$ \\
\hline$p$ & & $0.47^{b}$ & $0.14^{\mathrm{a}}$ & $0.20^{\mathrm{a}}$ & $0.10^{\mathrm{a}}$ & $0.47^{\mathrm{a}}$ \\
\hline
\end{tabular}

a: Mann-Whitney $U$ test, b: Pearson's $\chi^{2}$ test.

Table 3. Relationship between the Experience of Being Questioned by a Pharmacist while Showing the Drug Profile Book and the Patient's Behavior or Sense of Utility

\begin{tabular}{|c|c|c|c|}
\hline & \multicolumn{2}{|c|}{$\begin{array}{l}\text { Have you ever been } \\
\text { questioned by a pharmacist } \\
\text { while showing your drug } \\
\text { profile book? }\end{array}$} & \multirow[t]{2}{*}{$p$} \\
\hline & Yes $(n=84)$ & No $(n=148)$ & \\
\hline $\begin{array}{l}\text { Frequency of bringing the } \\
\text { drug profile book } \\
\qquad[\text { mean (S.D.) }]\end{array}$ & $2.6(1.4)$ & $2.2(1.7)$ & 0.02 \\
\hline $\begin{array}{l}\text { Frequency of the sticker } \\
\text { affix }[\text { mean (S.D.) }]\end{array}$ & $4.7(0.8)$ & $4.3(1.6)$ & 0.11 \\
\hline $\begin{array}{l}\text { Sense of utility } \\
{[\text { mean (S.D.) }]}\end{array}$ & $4.6(0.7)$ & $4.2(1.0)$ & $<0.01$ \\
\hline
\end{tabular}

Mann-Whitney $U$ test.

pharmacist while showing the drug profile book. Response rate of the "experience of showing the drug profile book to the physician" was significantly higher in the group with the experience of being questioned by a pharmacist while showing the drug profile book compared to the group without such experience (Table 4).

To the question of "whether the patient has ever shown the drug profile book to the physician", 86 patients "Yes", and 155 patients answered "No". Four patients were non-respondents. The sense of utility of the drug profile book and frequency of the sticker affix were significantly higher in the group which has the experience of showing the drug profile book to the physician (Table 5).

\section{DISCUSSION}

To the question of "whether the patient has experience of being questioned by a pharmacist while showing the drug profile book", only $34.3 \%$ of patients answered "Yes"; thus, pharmacists must improve patients' awareness of the drug profile book to provide safe medication in the future by strengthen-
Table 4. Relationship between the Experience of Being Questioned by a Pharmacist while Showing the Drug Profile Book and the Experience of Showing the Drug Profile Book to the Physician

\begin{tabular}{|c|c|c|c|c|}
\hline & & \multicolumn{2}{|c|}{$\begin{array}{l}\text { Have you ever shown } \\
\text { the drug profile book } \\
\text { to the physician? }\end{array}$} & \multirow[t]{2}{*}{ Total } \\
\hline & & Yes & No & \\
\hline \multirow{2}{*}{$\begin{array}{l}\text { Have you ever } \\
\text { been questioned by } \\
\text { a pharmacist while } \\
\text { showing your drug } \\
\text { profile book? }\end{array}$} & Yes & $40(47.6 \%)$ & $44(52.4 \%)$ & $84(100 \%)$ \\
\hline & No & $42(29.0 \%)$ & $103(71.0 \%)$ & $145(100 \%)$ \\
\hline Total & & 82 & 147 & 229 \\
\hline
\end{tabular}

Table 5. Relationship between the Experience of Showing the Drug Profile Book to the Physician and the Patient's Behavior or Sense of Utility

\begin{tabular}{llll}
\hline \hline & $\begin{array}{l}\text { Have you ever shown } \\
\text { the drug profile book } \\
\text { to the physician? }\end{array}$ & $p$ \\
\cline { 2 - 3 } & Yes $(n=86)$ No $(n=155)$ & 0.10 \\
\hline $\begin{array}{l}\text { Frequency of bringing the } \\
\text { drug profile book } \\
\quad \text { [mean (S.D.) })\end{array}$ & $2.5(1.5)$ & $2.2(1.6)$ & 0.01 \\
$\begin{array}{l}\text { Frequency of the sticker } \\
\text { affix [mean (S.D.) }\end{array}$ & $4.8(0.7)$ & $4.3(1.5)$ & \\
$\begin{array}{c}\text { Sense of utility } \\
\text { [mean (S.D.) })\end{array}$ & $4.7(0.6)$ & $4.2(1.0)$ & $<0.01$ \\
\hline Mann-Whitney $U$ test. & & &
\end{tabular}

ing medication consultation with patients using the drug profile book. The group with experience of being questioned by a pharmacist while showing the drug profile book scored higher on frequency of bringing the drug profile book, sense of utility, and frequency of the sticker affix, and they had a tendency to show the drug profile book to their physicians. We already found that frequency of bringing the drug profile book and frequency of the sticker affix rose in line 
with the strength of the sense of utility of the drug profile book. ${ }^{7)}$ In this paper, alongside confirmation of a relationship between i) the sense of utility of the drug profile book and ii) frequency of bringing the drug profile book, frequency of the sticker affix, and experience of showing the drug profile book to the physician, it is conceivable that the patients' sense of the utility of the drug profile book were related to the fact that the pharmacist communicated via the drug profile book and by the fact that patients themselves had more opportunity to utilize the drug profile book and that this, in turn, led to the patients safely undertaking medication. It was reported that $92.3 \%$ of physicians successfully prevented duplicate medication that may have occurred among medical facilities by using the drug profile book ${ }^{8)}$ while another report indicated that only $44.3 \%$ of patients showed the drug profile book at clinics or hospitals. ${ }^{9)}$ In the present survey, too, $35.7 \%$ of patients answered that they had shown the drug profile book to the physician, and, if pharmacists' actions of providing medication consultation using the drug profile book encourages patients to show the drug profile book to their physicians, duplicate medication or drug interaction can be prevented at drug prescription.

The limitations of this survey were as follows: participants were limited to patients receiving antihypertensives and regularly visiting the surveyed pharmacies, and the communication level including questions through the drug profile book was not closely investigated. Additionally, the survey has not provided any understanding of the relationship between the frequency with which a patient visits a pharmacy or medical facility and his or her perception and use of the drug profile book. Also, this survey result did not include such a case when the patient was questioned by a pharmacist based on the information in the drug profile book without showing it. However, we think the present survey is meaningful because we successfully suggested that pharmacists' actions of providing continuous medication consultation to patients using the drug profile book relates patients' awareness of the drug profile book and behavior regarding clinic/ hospital visits. Bringing communication between pharmacists and patients with other diseases than hypertension into view, we plan to continue the survey to examine the effect of the drug profile book on medication safety in near future.

Acknowledgments We express our deep gratitude for the cooperation of the company running pharmacies and the pharmacists working at those pharmacies.

Conflict of Interest The authors declare no conflict of interest.

\section{REFERENCES}

1) Japan Pharmaceutical Association. "Annual report of JPA.": 〈http://www.nichiyaku.or. jp/e/data/anuual_report2014e.pdf $\rangle$, cited 5 December, 2015.

2) Hayase Y., Yakugaku Zasshi, 123, 121-132 (2003).

3) Iijima H., Ishino Y., Ando H., Mogi H., Jpn. J. Pharm. Health Care Sci., 31, 223-227 (2005).

4) Japan Pharmaceutical Association. "Case Study of Drug Profile Book at the time of the Great East Japan Earthquake.’: 〈http:// www.nichiyaku.or.jp/action/wp-content/uploads $/ 2012 / 06 /$ shinsai_techo.pdf $\rangle$, cited 4 December, 2015.

5) Ojima F., Takeda N., Handa M., Nakagawa Y., J. Jpn. Soc. Hosp. Pharm., 43, 942-945 (2007) .

6) Watanabe H., Nakai Y., J. Jpn. Soc. Hosp. Pharm., 47, 559-562 (2011).

7) Shoji M., Onda M., Iwade K., Arakawa Y., Jpn. J. Pharm. Health Care Sci., 41, 139-146 (2015).

8) Yamaura K., Masuda M., Motohashi M., Kuramochi K., Ishizuka T., Someya T., Suganuma Y., Motohashi H., Yakugaku Zasshi, 123, 151-155 (2003).

9) Iijima H., Yamauchi K., Takahashi T., JJQSH, 5, 285-295 (2010). 